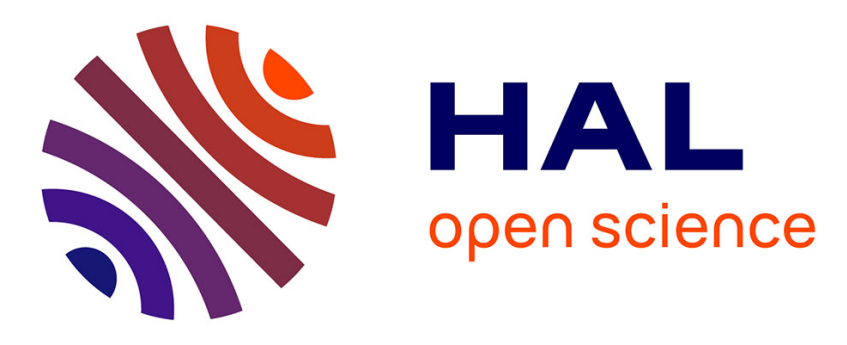

\title{
Vision Based UAV Attitude Estimation: Progress and Insights
}

Abd El Rahman Shabayek, Cédric Demonceaux, Olivier Morel, David Fofi

\section{To cite this version:}

Abd El Rahman Shabayek, Cédric Demonceaux, Olivier Morel, David Fofi. Vision Based UAV Attitude Estimation: Progress and Insights. Journal of Intelligent and Robotic Systems, 2012, 65, pp.295-308. 10.1007/s10846-011-9588-y . hal-00759018

\section{HAL Id: hal-00759018 https://hal.science/hal-00759018}

Submitted on 29 Nov 2012

HAL is a multi-disciplinary open access archive for the deposit and dissemination of scientific research documents, whether they are published or not. The documents may come from teaching and research institutions in France or abroad, or from public or private research centers.
L'archive ouverte pluridisciplinaire HAL, est destinée au dépôt et à la diffusion de documents scientifiques de niveau recherche, publiés ou non, émanant des établissements d'enseignement et de recherche français ou étrangers, des laboratoires publics ou privés. 


\title{
Vision Based UAV Attitude Estimation: Progress and Insights
}

\author{
Abd El Rahman Shabayek, Cédric Demonceaux, Olivier Morel, David Fofi
}

April 14, 2011

Le2i - UMR CNRS 5158

IUT Le Creusot

Université de Bourgogne, France

Abdelrahman.Shabayek@members.em-a.eu, Cedric.Demonceaux@u-

bourgogne.fr, Olivier.Morel@u-bourgogne.fr, David.Fofi@u-bourgogne.fr

\begin{abstract}
Unmanned aerial vehicles (UAVs) are increasingly replacing manned systems in situations that are dangerous, remote, or difficult for manned aircraft to access. Its control tasks are empowered by computer vision technology. Visual sensors are robustly used for stabilization as primary or at least secondary sensors. Hence, UAV stabilization by attitude estimation from visual sensors is a very active research area. Vision based techniques are proving their effectiveness and robustness in handling this problem. In this work a comprehensive review of UAV vision based attitude estimation approaches is covered, starting from horizon based methods and passing by vanishing points, optical flow, and stereoscopic based techniques. A novel segmentation approach for UAV attitude estimation based on polarization is proposed. Our future insightes for attitude estimation from uncalibrated catadioptric sensors are also discussed.
\end{abstract}

\section{Introduction}

In order to determine the pose of the vehicle accurately and rapidly, the regular approach is to use inertial sensors with other sensors and applying sensor fusion. Some sensors used for this purpose are the Global positioning sensor (GPS), inertial navigation sensor (INS), as well as other sensors such as altitude sensors (ALS) and speedometers. These sensors have some limitations. GPS sensor for example, is not available at some locations or readings subject to error. INS has the disadvantage of accumulation of errors. To overcome these limitations, vision-based navigation approaches have been developed. These approaches can be used where GPS or INS systems are not available or can be used with other sensors to obtain better estimations. UAV attitude estimation has been deeply studied in terms of data fusion of multiple low cost sensors in a Kalman filter (KF) framework to have the vehicle full state of position and orientation. But in pure vision based methods, if a horizontal world reference is visible (e.g horizon) the camera attitude can be obtained. 
In order to control a flying vehicle at least six parameters (pose of the vehicle) should be known; Euler angles representing the orientation of the vehicle and a vector of coordinates, representing the position of the vehicle. Pose estimation basically depends on viewing a world unchanging physical reference (e.g landmarks on the ground) for accurate estimation. Our main concern in this work is to review the work that focuses on attitude (roll, pitch, and yaw angles shown in figure (1) estimation rather than pose estimation.

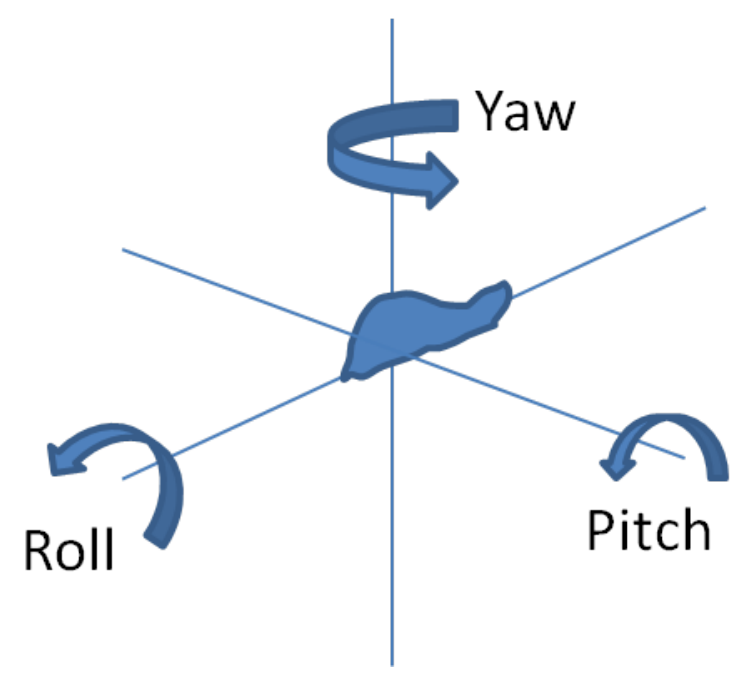

Figure 1: An illustrative sketch of the attitude (roll, pitch, and yaw angles)

In a typical flight, the demand for yaw angle will be largely constant and hence disturbances tend to have a relatively small effect on yaw. Further, small steady state errors are normally acceptable since (unlike roll and pitch) any errors will have no further effect on the UAV motion. Therefor, for the sake of UAV stabilization, the most important angles to be estimated are the pitch and roll angles as most of the work in literature propose. In this work, the focus will be on attitude estimation from perspective and omnidirectional cameras. It is intended to give a complete review with some views to enhance current work and propose novel ideas under investigation and development by our research group.

\subsection{Vision sensors for attitude estimation}

Vision based methods were first introduced by [1] . They proposed to equip a Micro Air Vehicle (MAV) with a perspective camera to have a vision-guided flight stability and autonomy system. Omnidirectional sensors for attitude estimation were first introduced by [2]. The omnidirectional sensors (Fisheye and Catadioptric cameras shown in figure (2)) were used in different scenarios. Catadioptric sensors are commercially available for reasonable prices. A catadioptric sensor has two main parts, the mirror and the lens. The lens could be telecentric or perspective. The sensor is in general assembled as shown in figure $2 \mathrm{c}$ ).

Omnidirectional sensors were used alone or in stereo configurations. Omnidirectional vision presents several advantages: a) a complete surrounding of the UAV can be 
captured and the horizon is totally visible, b) possible occlusions will have lower impact on the estimation of the final results, c) whatever the attitude of the UAV, the horizon is always present in the image, even partially, and the angles can always be computed, d) it is also possible to compute the roll and pitch angles without any prior hypothesis, contrary to the applications using a perspective camera. Yet, catadioptric vision also presents some drawbacks. For example,a) a catadioptric image contains significant deformations due to the geometry of the mirror and to the sampling of the camera, b) catadioptric cameras should be redesigned to a lower scale to be attached to a micro air vehicle (MAV).

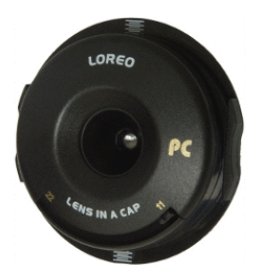

(a) Perspective

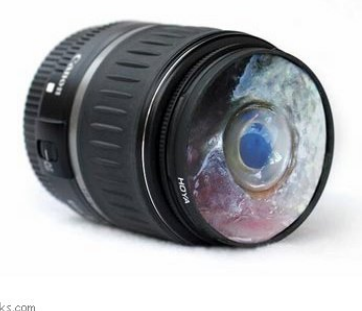

(b) Fisheye
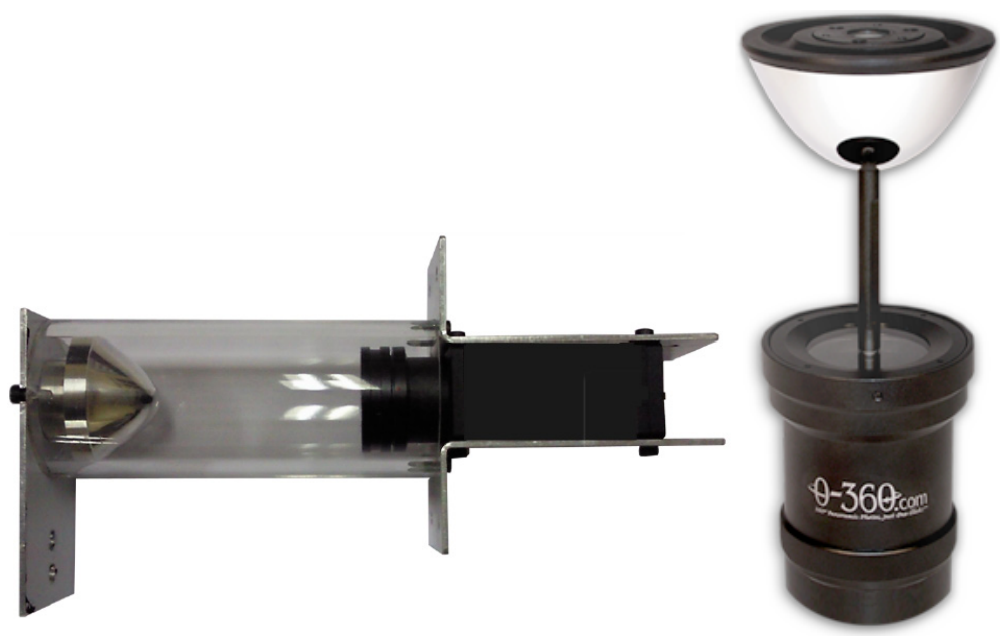

(c) Catadioptric

Figure 2: Perspective and omnidirectional (Fisheye and Catadioptric) cameras

\subsection{The main techniques for attitude estimation}

In literature, the first group of methods tries to detect a horizontal reference frame in the world to estimate the up direction and hence the attitude of the vehicle. The horizon, if visible, is the best natural horizontal reference to be used [1]. However, in urban environments the horizon might not be visible. Hence, the second group tries to find the vanishing points from parallel vertical and horizontal lines which are basic features of man made structure (e.g [3]). The third group was biologically inspired from insects, it employs the UAV motion (optical flow) for the sake of required estimation [4]. Stereo vision based techniques came to the play to open the door for more accurate estimation 
specially if combined with optical flow (e.g [5]). All these techniques will be discussed in the following sections.

Most of the employed techniques in literature use the Kalman filter (KF) or one of its variations in order to obtain an accurate and reliable estimation specially if more than one sensor is used and their measurements are fused. For a general parameter estimation issue, the extended Kalman filter (EKF) technique is widely adopted. Due to the processing of EKF in a linear manner, it may lead to sub-optimal estimation and even filter divergence. Nevertheless, state estimation using EKF assumes that both state recursion and covariance propagation are Gaussian. Unscented Kalman filter (UKF) resolves the nonlinear parameter estimation and machine learning problems. It can outperform the EKF especially for those highly nonlinear system dynamics/measurement processes. None of the Jacobean or derivatives of any functions are taken under the UKF processing [6]. For example in [7], using an EFK, the candidate horizon lines are propagated and tracked through successive image frames, with statistically unlikely horizon candidates eliminated. In [8], they followed the EKF framework to combine inertial and visual sensor for real time attitude estimation. They have designed a KF for image line measurements.

\subsection{Paper organization}

The paper will be organized as follows: sections $(2,3,4)$, will review the general techniques for attitude estimation from visual sensors (perspective and omnidirectional only) in detail. In section (2), horizon detection algorithms will be briefly explained and reviewed. Vanishing points based techniques are reviewed in section (3). The classical and hybrid approaches using stereo-vision and optical flow are reviewed in section (4). Finally we conclude in (5).

\section{Horizon Detection}

The visual sensor is not only a self-contained and passive like an INS but also interactive with its environment. An absolute attitude can be provided by detecting a reliable world reference frame. Attitude computation by vision is based on the detection of the horizon, which appears as a line in perspective images or a curve in omnidirectional images as shown in figure (3), and on the estimation of the angle between the horizon and a horizontal reference.

Due to the difficulty in obtaining ground-truth for aircraft attitude, most of the work in literature do not provide a quantitative measure of error in their estimates of roll and pitch. In [9], they provided a complexity and performance comparison between their method and other methods in litterature. They have included a comparison table of execution times for various published studies on visual attitude estimation.

In the following subsections, we will cover in detail the different segmentation approaches for horizon detection in section (2.1), a proposal to segment using polarization in section (2.2), and both the perspective and omnidirectional scenarios will be reviewed. Section (2.3) will briefly discuss horizon estimation and attitude computation in the perspective case. Section (2.4) will briefly discuss the same in the omnidirectional case specially in the catadioptric scenario which is frequently used. 


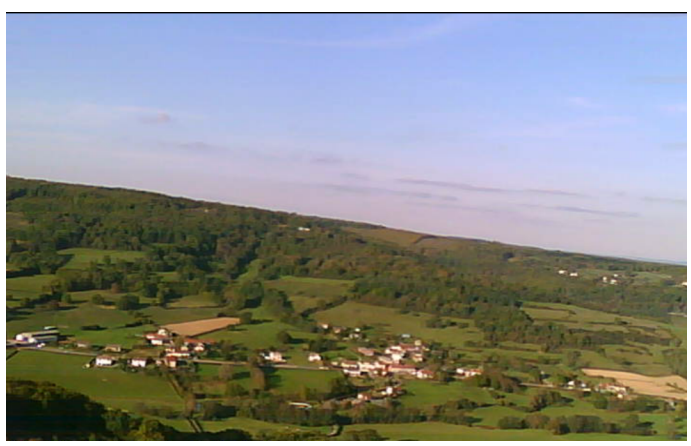

(a) Perspective

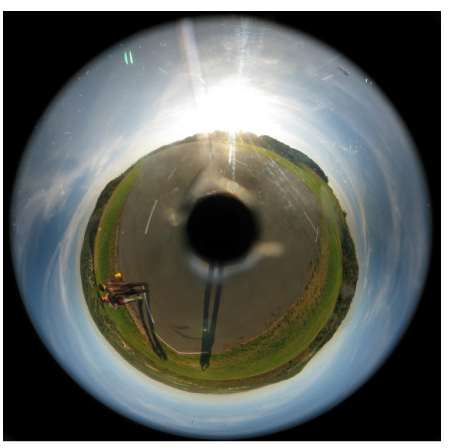

(b) Non-central catadioptric

Figure 3: Horizon in a) a perspective image, b) a non-central catadioptric image

\subsection{Sky/Ground Segmentation}

As the segmentation of sky and ground is a crucial step toward extracting the horizon line/curve, which is used for attitude estimation, these segmentation methods will be discussed here.

Using perspective vision, algorithms employing Gaussian assumptions for sky/ground segmentation fails in scenarios where the underlying Gaussian assumption for the sky and ground appearances is not appropriate [1]. These assumptions might be enhanced by a statistical image modeling framework by building prior models of the sky and ground then trained. Since the appearances of the sky and ground vary enormously, no single feature is sufficient for accurate modeling; as such, these algorithms rely both on color and texture as critical features. They may use hue and intensity for color representation, and the complex wavelet transform for texture representation. Then they may use Hidden Markov Tree models as underlying statistical models over the feature space [10]. In [7], the algorithm is based on detecting lines in an image which may correspond to the horizon, followed by testing the optical flow against the measurements expected by the motion filter.

Using omnidirectional vision, some algorithms use markovian formulation of sky/ground segmentation based on color information [2], or the sky/ground partitioning is done in the spherical image thanks to the optimization of the Mahalanobis distance between these regions. The search for points in either regions takes place in the RGB space [11]. In order to isolate the sky from the ground [12, 13], an approach based on the method employed by [14] weights the $R G B$ components of each pixel using the function $f(R G B)=3 B^{2} /(R+G+B)$.

In [9], they propose an algorithm which can be incorporated into any vision system (e.g. narrow angle, wide angle or panoramic), irrespective of the way in which the environment is imaged (e.g. through lenses or mirrors). The proposed horizon detection method consists of four stages: a) enhancing sky/ground contrast, b) determining optimum threshold for sky and ground segmentation, c) converting horizon points to vectors in the view sphere, and d) fitting 3D plane to horizon vectors to estimate the attitude.

In [15] they proposed segmentation using temperature from thermopile sensors in the thermal infrared band. However, in this work, the focus will be on attitude estimation from perspective and omnidirectional sensors only. 
The previous segmentation solutions are either complex and/or time consuming. A method based on polarization for segmentation in section (2.2) is proposed. We believe it will have significant enhancements in both complexity and time due to its simplicity . We propose a novel non-central catadioptric sensor where the mirror is a free-form shape and the camera is polarimetric (e.g FD-1665P Polarization Camera [16]) to be used for attitude estimation.

\subsection{Polarization based segmentation}

Instead of using color information or edge detection algorithms for segmentation which may require different complex models and offline processing as shown, we propose to use polarization information which exists in the surrounding nature. Polarization information are directly computed from three intensity images taken at three different angles of a linear polarization filter ( 0,45 , and 90 degrees) or at one shot using a polarimetric camera.

Using polarization for segmentation is not new. It was used for rough surface segmentation [17], material classification [18], water hazards detection for autonomous off-road navigation [19] , and similar applications. However, to the best of our knowledge, it is the first time to propose using polarization for sky/ground segmentation for UAV attitude estimation.

The most important polarization information are phase (angle) and degree. According to [18], the phase of polarization is computed as follows:

$$
\begin{aligned}
\theta \quad & =0.5 * \tan ^{-1}\left(\frac{I_{0}+I_{90}-2 I_{45}}{I_{90}-I_{0}}\right)+90 \\
\text { if } \quad I_{90}< & I_{0} \\
& \text { if } \quad I_{45}<I_{0} \\
& =\theta+90 \\
& \text { else } \quad \theta=\theta-90
\end{aligned}
$$

and the degree of polarization is:

$$
\phi=\frac{I_{90}-I_{0}}{\left(I_{90}+I_{0}\right) * \cos (2 \theta)}
$$

where $I_{0}, I_{45}$, and $I_{90}$ are intensity images taken at 0,45 , and 90 degrees of the rotating polarizer respectively (or at one shot from a polarimetric camera).

Figure (4) shows the segmentation results for non-central catadioptric images with the horizon detected by simply detecting the transition area. This technique is very simple and can be optimized by kind of binary search in the image having very rapid and robust results for the detected horizon in the image. Only few regions of the image are needed to be inspected for their degree or angle of polarization to decide for the search direction. Unlike conventional segmentation methods, thanks to polarization, we do not face the illumination problem caused by the sun being in the image.

In future work, we will provide detailed algorithms with complexity and run time comparison with other methods found in literature. 


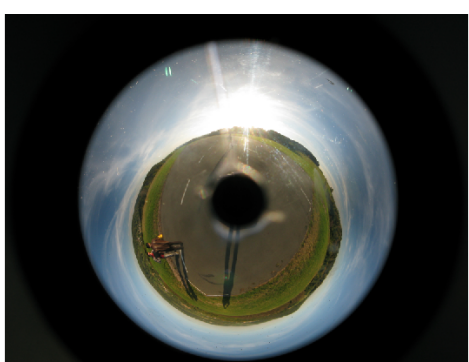

(a) 0 degree

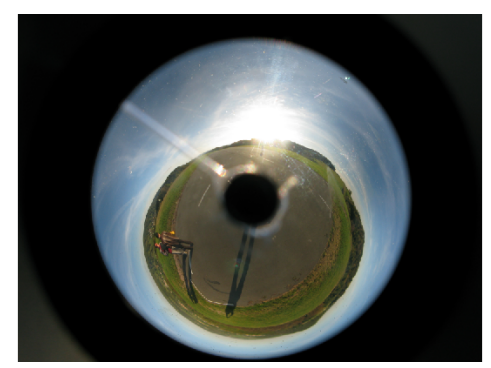

(b) 45 degree

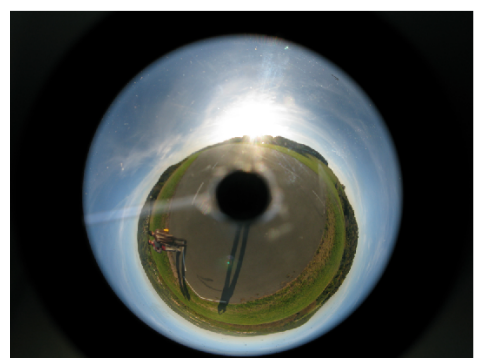

(c) 90 degree

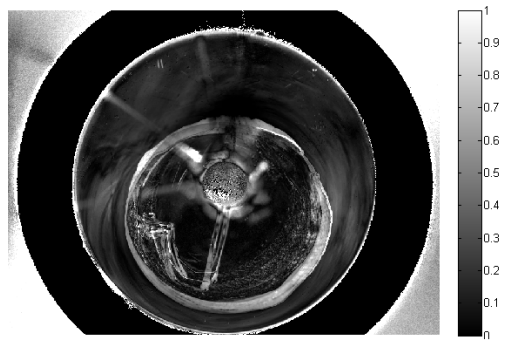

(d) Segmentation based on the de-(e) gree of polarization

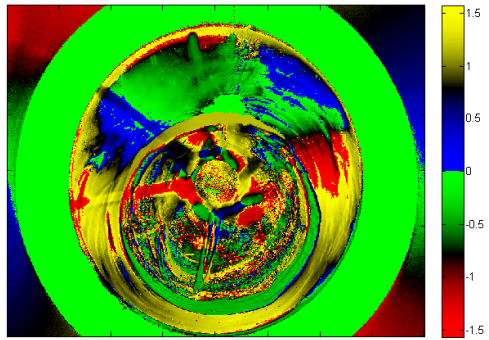

of polarization

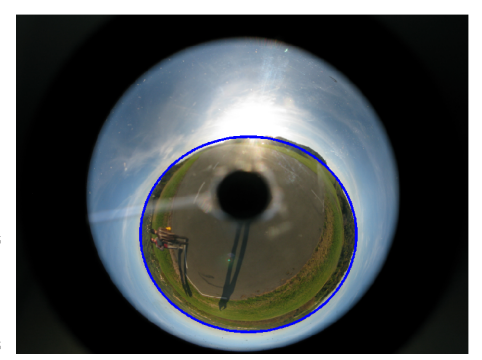

(f) Extracted horizon curve

Figure 4: Sky/Ground segmentation and horizon extraction based on polarization from non-central catadioptric images

\subsection{Using perspective sensors}

The horizon is projected as a line in the perspective image. Intuitively, it is required to extract that line. Most methods first segment the image into sky/ground areas, then take the separating points as the horizon line. The attitude is dependant on the gradient of that horizon line on the image plane. In literature, the general approach is to find the normal to the plane of the horizon in order to estimate the roll and pitch angles. The normal vector has direct mathematical relation with the attitude as expressed in different methods. The work done by [20, 21] are examples of successful autonomous control of a MAV based on attitude estimation from the horizon detected.

In literature, horizon detection problem has been addressed by segmentation and edge detection. In [1, 22] they proposed to equip a MAV with a perspective camera to have a vision-guided flight stability and autonomy system. They detected the horizon by extracting the straight line that separates the sky from the ground using the context difference of the two regions. In [10] they treated the horizon detection problem as a subset of image segmentation and object recognition, and used a percentage of the sky seen as an error signal to a flight stability controller on a MAV. The resulting system was stable enough to be safely flown by an untrained operator in real time. In contrast, [20] uses a direct edge-detection technique, followed by automatic threshold and a Hough-like algorithm to generate a "projection statistic" for the horizon. It claims a 99\% success rate over several hours of video. Importantly, it deals only with detection, not estimation of attitude. In [7] they propose an algorithm slightly similar to [20] in that it uses an edge detection technique followed by a Hough transform. However, they propose different image pre-filtering. In [23, 24, 25, 14] they use the centroids of sky and ground to ex- 
tract the horizon and derive the different angles. They try to simplify their work by using a circular mask to reduce image asymmetry and to simplify the calculations.

\subsection{Using omnidirectional sensors}

The use of a single perspective camera generates several drawbacks. Firstly, a partial view of the environment and important occlusions in the horizon can have a serious influence on the final result. Secondly, the horizon is visible only in a particular interval of roll and pitch values. If the UAV gets out of this interval, the final image is exclusively made of sky or earth and the horizon cannot be detected. Thirdly, it is only possible to compute the roll angle while the pitch is only approximated thanks to a hypothesis on the altitude of the UAV. All that pushed the need toward employing omnidirectional sensors to capture the horizon in almost all scenarios. The horizon appears as a curve in the omnidirectional image. It is common to use both fisheye and central catadioptric sensors. As both are treated by the equivalence sphere theory proposed by [26]. The particular geometric characteristics of the catadioptric sensor will be briefly explained in the next section. Once the horizon is detected, these characteristics are used to compute the attitude of the UAV.

\subsubsection{Central catadioptric projection of the horizon}

As demonstrated in [26], a 3D sphere projects on the equivalence sphere in a small circle, and then on the catadioptric image plane in an ellipse (see figure (5)). Consequently, the attitude computation is based on searching for an ellipse in the omnidirectional image or a small circle on the equivalent sphere which corresponds to the horizon. The geometrical properties of the equivalent sphere allow to deduce the roll and pitch angles. Indeed, the normal of the projected horizon on the sphere, which is also confounded with the line passing through the center of the sphere of equivalence and through the center of the earth represents in fact the attitude of the UAV depending on the position of the optical axis. Then, the computation of the coordinates of the optical axis is sufficient in order to deduce the roll and pitch angles.

\subsubsection{Horizon estimation and attitude computation}

To estimate the horizon, first the catadioptric image should be segmented to obtain the sky and ground and hence the points belonging to the horizon. Next, the horizon points should be back projected on the equivalence sphere. Finally, the best plane passes through the horizon on that sphere should be estimated to deduce its normal which gives the roll and pitch angles (e.g [2, 11] ).

In [2], they proposed to use an omnidirectional visual sensor in order to compute the attitude of a UAV. They have extended the work of [1, 22] to detect the curved horizon line. They show an adaptation of the Markov Random Fields (MRFs) to treat the deformations in the catadioptric images in order to detect the horizon and hence the catadiotric geometric characteristics are used to compute the UAV attitude. This method gives interesting results but do not use sufficiently the geometric characteristics of catadioptric vision. Moreover, the segmentation step is time consuming and do not permit a real time implementation. In [11], they present higher accuracy and computation time. They use 


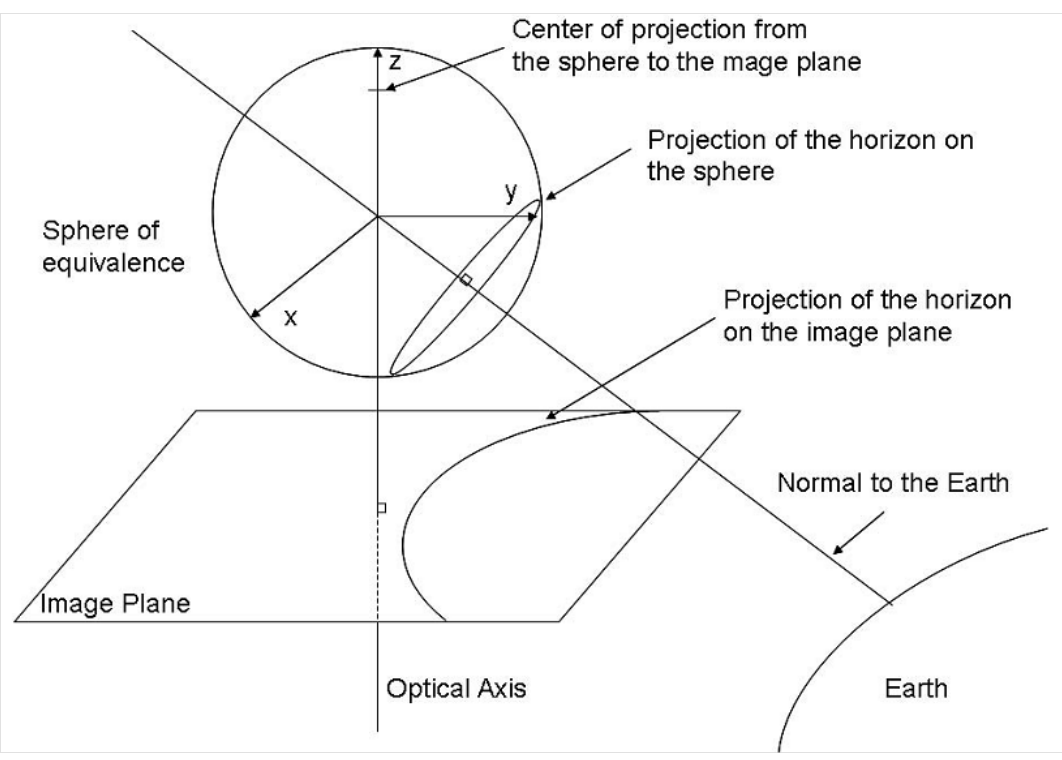

Figure 5: The relation between the horizon projection and the roll and pitch angles. (Adapted from [2]).

the geometric characteristics of the central catadioptric sensor for a formulation of the process as an optimization problem which is solved on the sphere of equivalence in order to compute directly the attitude angles. In [27], a hybrid method that is using the horizon and the homography is proposed. In [12, 13], they propose a similar approach to [2] for attitude estimation and a stereo-based system for height and motion estimation.

\section{Vanishing Points}

In [11, 2], the horizon was determined with Random Markow Fields or RGB based Mahalanobis distance. This approach requires the conditions where the horizon is visible (e.g low altitude in urban environments). In addition, it can not be used to estimate the yaw angle. In urban environments, the world reference can be the parallel lines which are a basic property of man-made structures. In this situations, vanishing points at the intersection of parallel vertical and horizontal lines can be used for attitude estimation (e.g [3]).

In [28], a batch process was developed to recover the history of camera orientations from non-linear optimization (bundle adjustment) of the vanishing points. In [8], their approach is based on vanishing points detection using raw line measurements directly to refine the attitude. They do not require any line tracking. But they fuse these line measurements with IMU gyro angle and compare each line segment with the current best attitude estimate.

Vanishing points were more exploited with the omnidirectional sensors. In [3], they use lines that are available in urban areas which avoids the limitations of horizon determination but it is still not possible to estimate the yaw angle, also it requires to determine the sky. Therefore, their approach is not suitable in dense city environments as well as closed areas. A more recent work proposes the use of vanishing points and infinite homography to estimate the helicopter attitude[29]. This approach can be used in urban environments, however this method has never been applied to a real UAV. In [30], they used the approach described in [29] to estimate helicopter attitude and improved it using 
a KF.

The research area in using vanishing points for attitude estimation is very active. It provides the intuitive solution for the attitude estimation problem specially in urban environments. Due to its importance, the following subsections will explain them in more details using perspective and omnidirectional sensors. For a comprehensive evaluation of several approaches for vanishing points detection, the reader is referred to [31, 32].

\subsection{Perspective}

The perspective projection of parallel lines intersects at a single point on an image called the vanishing point. In [33], given the camera calibration matrix, the geometric relationship between the vanishing points, the horizon, and camera orientation has been well established in a Gaussian sphere using 2D projective geometry . All vanishing point can be considered in a Gaussian sphere representation even those at infinity. For more details on representing vanishing points on a Gaussian sphere from a calibrated camera (see figure (6)), the reader is referred to [33, 34, 8].

\subsubsection{Gaussian sphere}

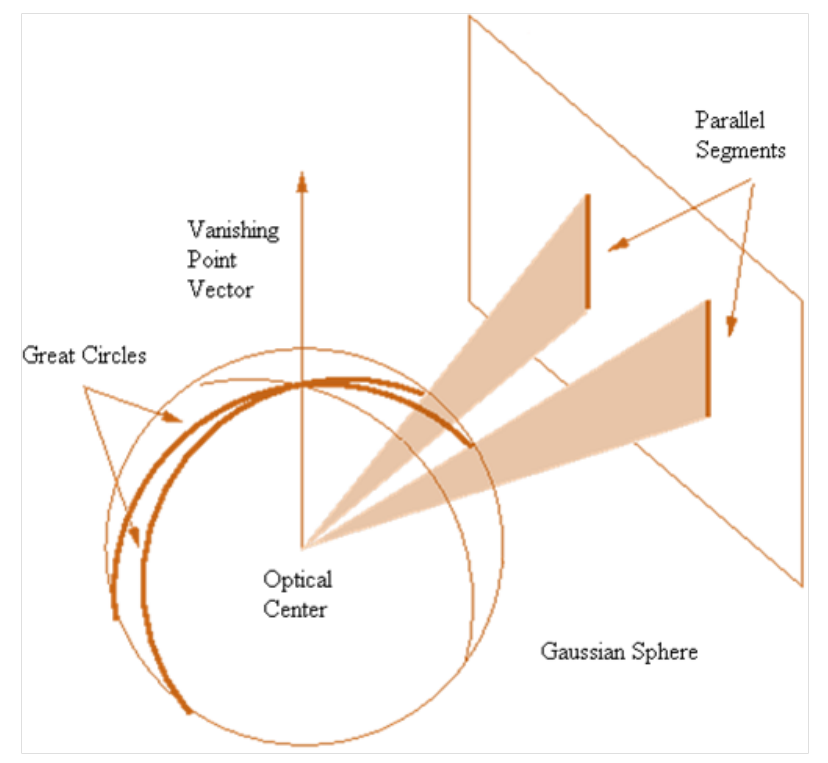

Figure 6: Gaussian Sphere adapted from [34]

The Gaussian sphere is a unit sphere which shares the same optical center of the pinhole camera. In the $2 \mathrm{D}$ projective space, an image line is represented as a normal vector of a great circle in homogeneous coordinates. The intersection of two parallel edges is a vanishing point which can be computed by the duality between the points and lines in a projective plane $i . e v_{i j}=l_{i} \times l_{j}$ where $v_{i j}$ is a vanishing point and $l_{i}$, and $l_{j}$ are parallel lines. The vanishing point is the direction to the corresponding 3D point at infinity.

In a calibrated camera, the vanishing points formed by vertical edges and those formed by horizontal edges are geometrically constrained to:

$$
v_{\text {vertical }}^{T} \cdot v_{\text {horizontal }}^{i}=0, i=1, \ldots ., n \text {. }
$$


Vanishing points that lie on the same plane define a vanishing line in an image. Then the horizon is equal to the vanishing line that links any two horizontal vanishing points. The horizon is dual to the vertical vanishing point. This can be geometrically explained as having the horizon as the projection of the world ground plane, and the normal to the ground plane is projected on the vertical vanishing point i.e:

$$
\text { horizon }=v_{\text {horizontal }}^{i} \times v_{\text {horizontal }}^{j}
$$

The UAV attitude can be determined when either the vertical vanishing point or at least two horizontal vanishing points are recovered from the image given that a) the great circle in the Gaussian sphere has the same orientation as the world ground plane, and b) the relative camera pose with respect to an UAV is known. In general, it is assumed that the camera is attached to the UAV where the camera's principle axis is aligned along the UAV centerline.

\subsubsection{Vertical vanishing points}

In urban environments, vertical edges meet at a single vanishing point in the same direction as the gravity in the world coordinates. The vertical vanishing point is the perspective projection of the world z-axis with the camera pose matrix. Let $v_{v e r t i c a l}=(v x, v y)^{T}$, be the vertical vanishing point, then once it is found, the attitude can be immediately computed by (see figure (7)):

$$
\text { roll }=\phi=\operatorname{atan} 2(v x, v y), \text { pitch }=\theta=\operatorname{atan} \frac{1}{\sqrt{v x^{2}+v y^{2}}} .
$$

The horizon line on the image is a line defined by the vertical vanishing point where:

$$
\left.\left(\sin _{\phi} \cos _{\theta}\right) x+\left(\cos _{\phi} \cos _{\theta}\right)\right) y+\sin _{\theta}=0 .
$$

\subsubsection{Horizontal vanishing points}

In urban environments, horizontal edges which are orthogonal to the gravity direction meet at vanishing points in the world ground plane (see figure (8)). One of the horizontal vanishing points is the perspective projection of the world $\mathrm{x}$-axis with the camera pose matrix. Then the horizontal vanishing point is:

$$
v_{\text {horizontal }}=\left[\frac{\cos _{\phi} \sin _{\psi}-\sin _{\phi} \sin _{\theta} \cos _{\psi}}{\cos _{\theta} \cos \psi}, \frac{-\sin _{\phi} \sin _{\psi}-\cos _{\phi} \sin _{\theta} \cos _{\psi}}{\cos _{\theta} \cos _{\psi}}\right]^{T}
$$

where $\psi$ is the yaw angle. All the horizontal vanishing points are along the horizon and their locations are determined by the different yaw angles. 


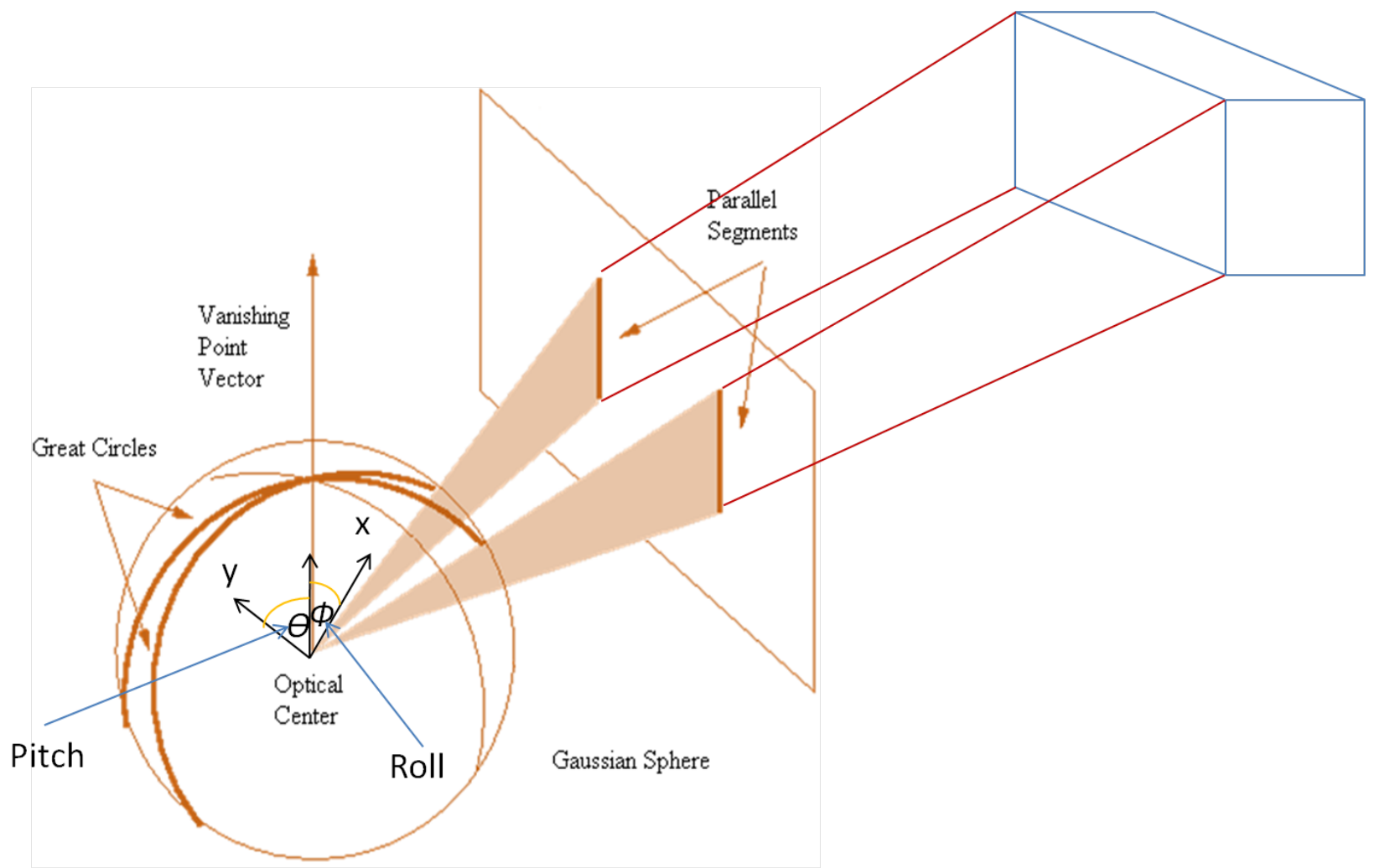

Figure 7: Illustration of the relation between a vertical vanishing point and the roll and pitch angles.

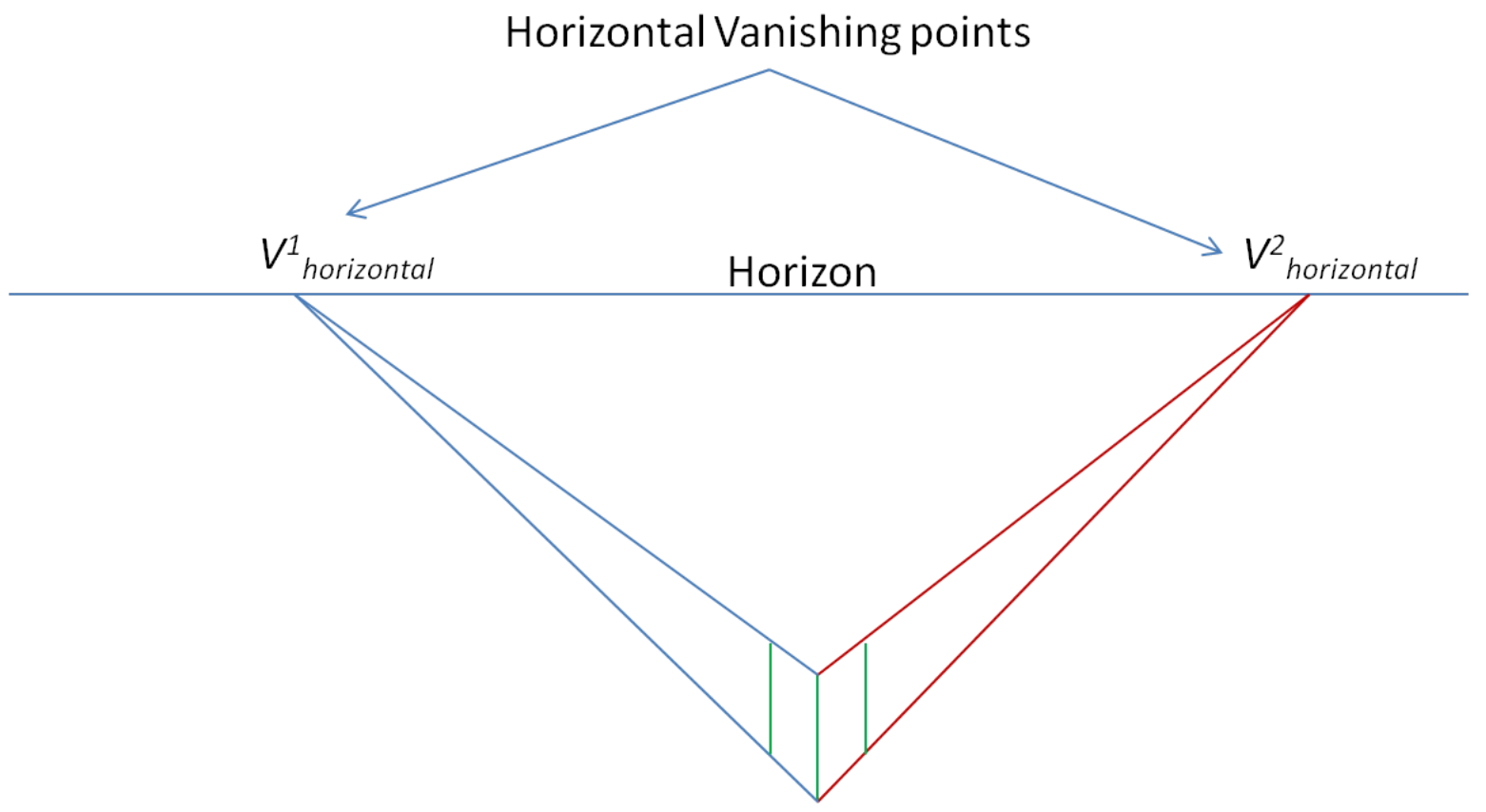

Figure 8: Horizontal vanishing points. 


\subsection{Catadioptric}

As previously mentioned, Projection of $3 \mathrm{D}$ world points to the image plane can be done in three steps. Firstly the point is projected to the equivalent sphere, then to the plane at infinity and finally to the image plane. Besides, projection of 3D lines generates a great circle on the equivalent sphere (see figure (5)). By back projecting every candidate edge on the sphere and checking each edge if it verifies the great circle constraint, one can decide which edges belong to real 3D lines. In order to do this, the edges divided according to their gradient orientations and selected by their lengths are back projected to the sphere. Then plane normal of the great circle is computed by cross product of first and last edgel directions. In addition, parallel lines have the same vanishing direction on the equivalent sphere. Therefore, dominant parallel lines can be extracted by counting lines which satisfy some similarity threshold based on their vanishing direction. By excluding found parallel lines and repeating the same algorithm, these dominant vanishing directions can be found. Based on an orthogonality threshold, if $\left|u_{1} \times u_{2}\right| \leq$ OrthogonalityThreshold, the cross product $u_{3}=u_{1} \times u_{2}$ is computed to determine the third vanishing direction, where $u_{i}$ s are orthogonal parallel lines. If the inequality is not satisfied, this means that the detection of orthogonal parallel lines is failed; therefore attitude estimation at that frame should be skipped. In that case, it is thought that the UAV does not change its orientation.

\section{Stereo Vision And Optical Flow}

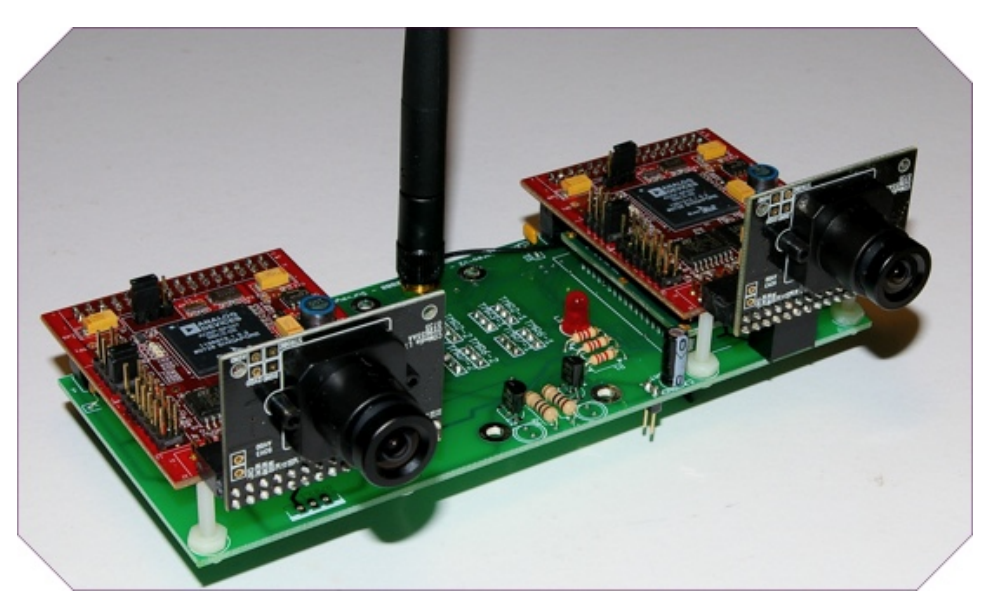

(a) Stereo vision System

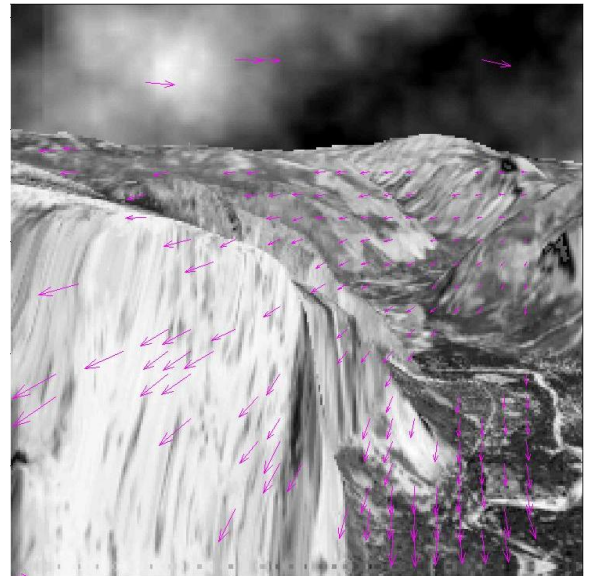

(b) Phase-based estimation of the optical flow field adapted from [35]

Figure 9: Stereo Vision and Optical Flow

\subsection{Stereo vision}

Computer stereo vision, is a part of computer vision where two cameras capture the same scene but they are separated by a distance as shown in figure (9a). A computer compares 
the images while shifting the two images together over top of each other to find the parts that match. The shifted amount is called the disparity.

In [36], the authors used a dual CCD stereo vision system in order to improve the computation of the attitude by determining the complete pose of the UAV taking advantages of UKF. However, this system relies on the capture of ground targets/landmarks in both images which limits the environment in which the UAV can move. In [37], they presented a mixed stereoscopic vision system made of fish-eye and perspective cameras for altitude estimation. Since there exists a homography between the two captured views, where the sensor is calibrated and the attitude is estimated by the fish-eye camera using the techniques in [2, 3], the algorithm searches for the altitude which verifies this homography. It allows real time implementation. In [12, 13] , the conventional stereo system was used for altitude computation. But for attitude computation, they also used a similar approach to [2].

\subsection{Optical flow}

Optical flow is the approximation of the motion field which can be computed from timevarying image sequences (see figure (9b)). It provides many important visual cues [38]. It is possible to estimate the flight altitude from the observed optical flow in the downward direction. Faster optic flow indicates a low flight altitude. Obstacles can be detected in the forward direction by detecting expansion, or divergence, in the forward visual field.

Optical Flow Estimation Methods are based on a) differential Techniques (dense motion field) where spatial and temporal variations of the image brightness at all pixels are considered, b) phase methods where response of filters to energy signals are used, c) matching techniques (sparse motion field) where the disparity of special image points (features) between frames is estimated.

In [39, 40], they derived a form of the KF that uses the relationship between visionbased measurements and the motion of the camera. The resulting implicit extended Kalman filter (IEKF) can be used to recover the camera motion states. In [41], they reused [39, 40] work in terms of an aircraft state-estimation problem by incorporating aircraft dynamics into the IEKF framework. The resulting formulation partially estimated the aircraft states but exhibited relatively slow convergence. Improvements have been demonstrated by [42, 43] who also used an aircraft model. Unfortunately, accurate MAV models are often not available within an aggressive flight regime where the aerodynamics are difficult to characterize.

Several techniques have utilized the kinematic relationship between camera motion and the resulting optical flow to directly solve for unknown motion parameters using constrained optimization. In [44, 45, 46], these techniques depend on at least partial knowledge of the translational velocity for use in the optimization. This knowledge often depends on GPS measurements. In [47], they addressed the problem of estimating aircraft states during a GPS-denied mission segment. An iterative optimization approach is adopted to determine the angular rates and the wind-axis angles. No knowledge of vehicle velocity is required. The coupled aircraft-camera kinematics are used to solve for aircraft states in similar fashion to previous efforts; however, velocity dependencies are removed through decoupling the optical flow resulting from angular and translational motion, respectively. Angular rate estimates are obtained initially and used to setup a simple linear least-squares problem for the aerodynamic angles. Performance of the least-squares problem is further improved through the application of a weighting scheme 
derived from parallax measurements.

But Optical flow is inherently noisy, and obtaining dense and accurate optical flow images is computationally expensive. Additionally, systems that rely on optical flow for extracting range information need to discount the components of optical flow that are induced by rotations of the aircraft, and use only those components that are generated by the translational motion of the vehicle. This either requires an often noisy, numerical estimate of the roll, pitch, and yaw rates of the aircraft, or additional apparatus for their explicit measurement, such as a three-axis gyroscope. Furthermore, the range perceived from a downward facing camera or optical flow sensor is only dependent upon altitude, velocity, and the aircraft's attitude [48].

Stereo vision provides an attractive approach to solve some of the problems of providing guidance for autonomous aircraft operating in low-altitude or cluttered environments [5, 48]. In [7], the optical flow of the image for each candidate horizon line is calculated, and using these measurements from the perspective camera, they are able to estimate the body rates of the aircraft. In [49], they estimate the heading of a small fixed pitch four rotor helicopter. Heading estimates are computed using the optical flow technique of phase correlation on images captured using a down facing camera. The camera is fitted with an omnidirectional lens and the images are transformed into the log-polar domain before the main computational step.

\subsection{Optical flow from stereo vision}

In [5, 48, 50], they proposed a stereo vision system from two non-central catadioptric cameras. The profile of the mirror is designed to ensure that equally spaced points on the ground, on a line parallel to the camera's optical axis, are imaged to points that are equally spaced in the camera's image plane. However, they have not used physical mirrors, but instead used high resolution video cameras equipped with wide-angle fisheye lenses and simulated the imaging properties of the mirrors by means of software lookup tables. Given the measured disparity surface from the optical flow, the attitude (roll and pitch) and altitude can be estimated by iteratively fitting the modelled surface to the measurements. They propose to enhance their method by estimating attitude and altitude with respect to an assumed ground plane by reprojecting the disparity points into $3 \mathrm{D}$ coordinates. In [51], he presentes a technique for estimating the aerodynamic attitude in the presence of dynamic obstacles. This technique relies on optical flow and stereo vision to remove dynamic objects from the static background. The resulting flow field is used for attitude computation from the calculated flow centroids.

\section{Conclusion}

Any UAV may fly in low, middle, or high altitudes. We believe that the Omnidirectional sensors should be always used because either the horizon will be always visible (middle and high altitudes) or the vanishing points directions in low altitudes. If the horizon is visible, then attitude should be estimated based on it. We proposed a simpler method for segmentation and horizon detection based on polarization which can be used. In urban environments, techniques based on vanishing points should be used. If obstacle 


\begin{tabular}{|c|c|c|c|}
\hline Papers & Roll & Pitch & Yaw \\
\hline \multicolumn{4}{|l|}{ Horizon detection } \\
\hline$[1,22,23,24,25,14,11,2,7,9]$ & $\mathrm{X}$ & $\mathrm{X}$ & \\
\hline$[27,12,13]$ & $\mathrm{X}$ & $\mathrm{x}$ & $\mathrm{x}$ \\
\hline \multicolumn{4}{|l|}{ Vanishing points } \\
\hline$[3,8]$ & $\mathrm{X}$ & $\mathrm{X}$ & \\
\hline$[29,30]$ & $\mathrm{X}$ & $\mathrm{X}$ & $\mathrm{X}$ \\
\hline \multicolumn{4}{|l|}{ Stereo vision and optical flow } \\
\hline$[46,47,7,5,48,50,51,37]$ & $\mathrm{X}$ & $\mathrm{X}$ & \\
\hline$[12,13]$ & $\mathrm{X}$ & $\mathrm{X}$ & $\mathrm{X}$ \\
\hline
\end{tabular}

Table 1: The estimated attitude angles (Roll, Pitch, and Yaw).

avoidance and altitude estimation are required with attitude estimation, then optical flow approaches from stereoscopic sensors are recommended.

In the work presented in sections (2.4,3), the catadioptric sensors used were assumed to be central sensors having a single view point. But in practice, non-central catadioptric sensors are more practical due to higher resolution and simplicity in design. Even the claimed central sensors, might be slightly non-central due to possible misalignments of the lens. All of that pushed the need toward developing methods for attitude estimation from non-central catadioptric sensors.

Currently, we develop an approach for UAV attitude estimation from uncalibrated non-central catadioptric sensor with unknown mirror shape. We assume that the mirror is a symmetric surface of revolution (SOR), the catadioptric image has two quadrics (inner and outer cross sections) as shown in figure (4), and a horizontal reference is visible in the captured image (e.g horizon, horizontal edges). We try to find the angle between the axis of revolution and the normal of the plan containing the horizontal reference.

In summary, a comprehensive review on attitude estimation approaches from visual sensors has been covered. Table (1) shows the papers and the estimated angles in the reviewed work. The main general approaches has been shortly discussed. Horizon detection which is the main key for attitude estimation in middle and high altitudes, has been discussed in the light of current ongoing work using different visual sensors. The sky/ground segmentation methods, for horizon detection, found in literature have been reviewed and a novel approach based on polarization applied to UAV attitude estimation has been proposed. In low altitudes the horizon is mostly invisible, hence the line segments found in man-made structures are exploited to obtain vanishing points for attitude computations. Stereoscopic and optical flow based techniques have been also covered. Optical flow computation from stereoscopic systems is proposed in very recent works. To the best of our knowledge, the main work done for UAV attitude estimation from vision sensors (perspective and omnidirectional only) has been covered here.

\section{References}

[1] Scott M. Ettinger, Michael C. Nechyba, Peter G. Ifju, and Martin Waszak. Visionguided flight stability and control for micro air vehicles. In IEEE/RSJ Int Conf on Robots and Systems, pages 2134-2140, 2002. 
[2] Cédric Demonceaux, Pascal Vasseur, and Claude Pégard. Omnidirectional vision on uav for attitude computation. In ICRA, pages 2842-2847, 2006.

[3] Cédric Demonceaux, Pascal Vasseur, and Claude Pégard. Uav attitude computation by omnidirectional vision in urban environment. In ICRA, pages 2017-2022, 2007.

[4] Geoffrey L. Barrows, Javaan S. Chahl, and Mandyam V. Srinivasan. Biomimetic visual sensing and flight control. In Proceedings Seventeenth International Unmanned Air Vehicle Systems Conference, 2002.

[5] Richard J. D. Moore, Saul Thurrowgood, Daniel Bland, Dean Soccol, and Mandyam V.Srinivasan. A stereo vision system for uav guidance. In IEEE/RSJ International Conference on Intelligent Robots and Systems, 2009.

[6] Shan-Chih Hsieh, L.K. Wang, Fei-Bin Hsaio, Kou-Yuan Huang, and Fan-Jen Tsai. Airborne attitude/ground target location determinations using unscented kalman filter. In Aerospace Conference, 2004. Proceedings. 2004 IEEE, volume 3, pages 6 vol. (xvi+4192), 2004.

[7] Damien Dusha, Wageeh Boles, and Rodney Walker. Attitude estimation for a fixedwing aircraft using horizon detection and optical flow. In Proceedings of the 9th Biennial Conference of the Australian Pattern Recognition Society on Digital Image Computing Techniques and Applications, DICTA '07, pages 485-492, Washington, DC, USA, 2007. IEEE Computer Society.

[8] Myung Hwangbo and Takeo Kanade. Visual-inertial attitude estimation using urban scene regularities. In To appear in IEEE International Conference on Robotics and Automation, 2011.

[9] Saul Thurrowgood, Dean Soccol, Richard J. D. Moore, Daniel Bland, and Mandyam V. Srinivasan. A vision based system for attitude estimation of uavs. In IEEE/RSJ International Conference on Intelligent Robots and Systems, 2009.

[10] Sinisa Todorovic and Michael C. Nechyba. Sky/ground modeling for autonomous mav flight. In In IEEE International Conference on Robotics and Automation (ICRA, pages 1422-1427, 2003.

[11] Cédric Demonceaux, Pascal Vasseur, and Claude Pégard. Robust attitude estimation with catadioptric vision. In IROS, pages 3448-3453, 2006.

[12] Iván F. Mondragón, MiguelA. Olivares-Méndez, Pascual Campoy, Carol Martínez, and Luís Mejias. Unmanned aerial vehicles uavs attitude, height, motion estimation and control using visual systems. Autonomous Robots, 29, 2010.

[13] Iván F. Mondragón, Pascual Campoy, Carol Martinez, and Miguel Olivares. Omnidirectional vision applied to unmanned aerial vehicles uavs attitude and heading estimation. Robotics and Autonomous Systems, March 2010.

[14] T.D.Cornall, G.K.Egan, and A.Price. Aircraft attitude estimation from horizon video. ELECTRONICS LETTERS, 42(12), June 2006.

[15] B. Taylor, C. Bil, and S. Watkins. Horizon sensing attitude stabilisation: A vmc autopilot. In 18th International UAV Systems Conference, 2003. 
[16] Fd-1665p polarization camera, http://www.fluxdata.com/products/fd-1665polarization-camera/.

[17] Patrick Terrier, Vincent Devlaminck, and Jean Michel Charbois. Segmentation of rough surfaces using a polarization imaging system. J. Opt. Soc. Am., pages $423-$ 430, 2008.

[18] L. B. Wolff. Polarization-based material classification from specular reflection. IEEE Trans. Pattern Anal. Mach. Intell., 12:1059-1071, November 1990.

[19] Bin Xie, Zhiyu Xiang, Huadong Pan, and Jilin Liu. Polarization-based water hazards detection for autonomous off-road navigation. In Intelligent Robots and Systems, 2007. IROS 2007. IEEE/RSJ International Conference on, pages 3186 -3190, 292007-nov.2 2007.

[20] G. Bao, Z. Zhou, S. Xiong, X. Lin, , and X.Ye. Towards micro air vehicle flight autonomy research on the method of horizon extraction. In Instrumentation and Measurement Technology Conference, . IMTC '03. Proceedings of the 20th IEEE, 2003., 2003.

[21] S. Todorovic and M. C. Nechyba. A vision system for intelligent mission profiles of micro air vehicles. IEEE Transactions on Vehicular Technology, 53:1713-1725, 2004.

[22] Scott M. Ettinger, Michael C. Nechyba, Peter G. Ifju, and Martin Waszak. Visionguided flight stability and control for micro air vehicles. Advanced Robotics, 17(7):617-640, November 2003.

[23] Terry D. Cornall and G. K. Egan. Measuring horizon angle from video on a small unmanned air vehicle. In 2nd International Conference on Autonomous Robots and Agents, 2004.

[24] T. D. Cornall and G. K. Egan. Measuring horizon angle from video on a small unmanned airborne vehicle. In 2nd International Conference on Autonomous Robots and Agents, Palmerston North, New Zealand, 2004.

[25] Terry D.Cornall and G.K.Egan. Measuring horizon angle from video on a small unmanned air vehicle. Technical report, MONASH University, Department of Electrical and Computer Systems Engineering, 2005.

[26] S. Baker and S. K. Nayar. A theory of catadioptric image formation. In International Conference on Computer Vision (ICCV03), pages 1351-1358, Oct 2003.

[27] J.C.Bazin, I.S.Kweon, C.Demonceaux, and P.Vasseur. Uav attitude estimation by combining horizon-based and homography-based approaches for catadioptric image. In 6th IFAC/EURON Intelligent Autonomous Vehicles (IAV07), Toulouse, France, 2007.

[28] M.E. Antone and S. Teller. Automatic recovery of relative camera rotations for urban scenes. pages II: 282-289, 2000. 
[29] Jean Charles Bazin, Inso Kweon, Cédric Demonceaux, and Pascal Vasseur. Uav attitude estimation by vanishing points in catadioptric images. In ICRA, pages 2743-2749, 2008.

[30] Metin Tarhan and Erdinc Altug. Ekf based attitude estimation and stabilization of a quadrotor uav using vanishing points in catadioptric images. Journal of Intelligent \&amp; Robotic Systems, pages 1-21-21, September 2010.

[31] J. A. Shufelt. Performance evaluation and analysis of vanishing point detection techniques. Pattern Analysis and Machine Intelligence, IEEE Transactions on, 21(3):282-288, 1999.

[32] Patrick Denis, James H. Elder, and Francisco J. Estrada. Efficient edge-based methods for estimating manhattan frames in urban imagery. In Proceedings of the 10th European Conference on Computer Vision: Part II, pages 197-210, Berlin, Heidelberg, 2008. Springer-Verlag.

[33] S.T.Barnard. Interpreting perspective images. Artificial Intelligence, 21:435-462, 1984.

[34] Carsten Rother. A new approach for vanishing point detection in architectural environments. In In Proc. 11th British Machine Vision Conference, pages 382-391, 2000 .

[35] http://www.mathworks.com/matlabcentral/fileexchange/2422-phase-basedoptical-flow.

[36] L.K. Wang, S.-C. Hsieh, E.C.-W. Hsueh, Fei-Bin Hsaio, and Kou-Yuan Huang. Complete pose determination for low altitude unmanned aerial vehicle using stereo vision. In Intelligent Robots and Systems, 2005. (IROS 2005). 2005 IEEE/RSJ International Conference on, pages 108 - 113, 2005.

[37] D. Eynard, P. Vasseur, C. Demonceaux, and V. Fremont. Uav altitude estimation by mixed stereoscopic vision. In Intelligent Robots and Systems (IROS), 2010 IEEE/RSJ International Conference on, pages 646 -651, 2010.

[38] J.J GIBSON. The ecological approach to visual perception. Houghton Mifflin, Boston, 1950.

[39] S. Soatto, R. Frezza, and P Perona. Motion estimation via dynamic vision. IEEE Transactions on Automatic Control, 41:393-413, 1996.

[40] S. Soatto and P. Perona. Recursive 3-d visual motion estimation using subspace constraints. International Journal of Computer Vision, 22:235-259, 1997.

[41] P. Gurfil and H. Rotstein. Partial aircraft state estimation from visual motion using the subspace constraints approach. Journal of Guidance, Control, and Dynamics, 24:1016-1028, 2001.

[42] T. Webb, R. Prazenica, A. Kurdila, and R. Lind. Vision-based state estimation for autonomous micro air vehicles. Proc. of the AIAA Guidance, Navigation, and Control Conference, page 5249, 2004. 
[43] T. Webb, R. Prazenica, A. Kurdila, and R. Lind. Vision-based state estimation for uninhabited aerial vehicles. Proc. of the AIAA Guidance, Navigation, and Control Conference, page 5869, 2005.

[44] G. Gebert, D. Snyder, J. Lopez, N. Siddiqi, and J. Evers. Optical flow angular rate determination. Proc. of the International Conference on Image Processing, 1:49-52, 2003.

[45] R.V. Iyer, Z. He, and P.R. Chandler. On the computation of the ego-motion and distance to obstacles for a micro air vehicle. Proc. of the IEEE American Control Conference, 2006.

[46] J. Kehoe, R. Causey, A. Arvai, and R. Lind. Partial aircraft state estimation from optical flow using non-model-based optimization. Proc. of the IEEE American Control Conference, 2006.

[47] Joseph J. Kehoe, AdamS. Watkins, Ryan S. Causey, and Rick Lind. State estimation using optical flow from parallax-weighted feature tracking. Proceedings of the AIAA Guidance, Navigation, and Control Conference, 2006.

[48] Richard J. D. Moore, Saul Thurrowgood, Dean Soccol, Daniel Bland, and Mandyam V. Srinivasan. A bio-inspired stereo vision system for guidance of autonomous aircraft. Advances in Theory and Applications of Stereo Vision, 2010.

[49] John Stowers, Andrew Bainbridge-Smith, Michael Hayes, and Steven Mills. Optical flow for attitude estimation of a quadrotor helicopter. European Micro Air Vehicle Conference, 2009.

[50] Richard J. D. Moore, Saul Thurrowgood, Daniel Bland, Dean Soccol, and Mandyam V.Srinivasan. Uav altitude and attitude stabilisation using a coaxial stere ovision system. In IEEE International Conference on Robotics and Automation, 2010.

[51] Chris Hedden. Vision-based uav aerodynamic attitude estimation in the presence of dynamic obstacles. University of Kansas, 122010. 